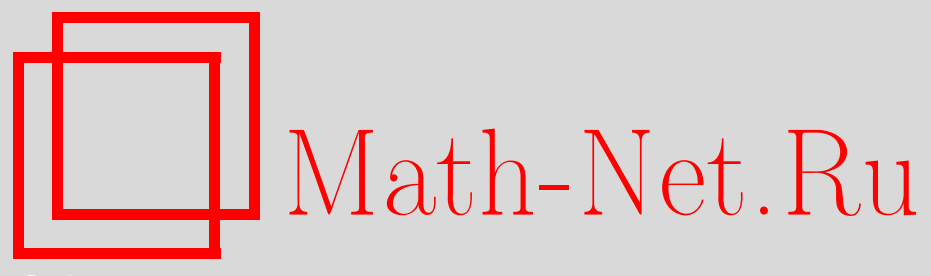

В. Клепцын, Игры Джона Конвея, Квант, 2020, номер 5, 1014

DOI: https://doi.org/10.4213/kvant20200502

Использование Общероссийского математического портала Math-Net.Ru подразумевает, что вы прочитали и согласны с пользовательским соглашением http: //www.mathnet.ru/rus/agreement

Параметры загрузки:

IP: 54.147 .182 .235

26 апреля 2023 г., 14:51:17

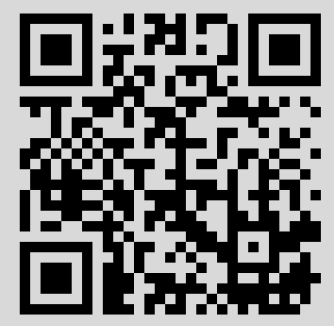




\section{Игры Джона Конвея}

\section{В.КЛЕПЦЫН}

Д жон Конвей (26.12.1937-11.04.2020) говорил, что не проработал ни единого дня, а только играл. Блестящий математик современности, он работал «играючи» во многих областях, и мне хочется вспомнить часть того, чем он занимался и запомнился.

\section{Игра «Жизнь»}

Пожалуй, самое широко известное открытие Конвея - это придуманная им игра «Жизнь». Устроена она так: на (бесконечной) бумаге в клеточку часть клеток объявляется «живыми» (или «заполненными», и обычно их закрашивают), а остальные «мертвыми» (или «пустыми»). После этого их конфигурация начинает (вся одновременно!) эволюционировать по следующим исключительно простым правилам:

1. Если у живой клетки в некоторый момент из 8 соседей было 2 или 3 живые клетки, то на следующий ход она выживает, в противном случае она умирает (обычно говорят, что если живых соседей 1 или 0 , то она умирает «от одиночества», а если больше 3, то «от перенаселения»).

2. Если у пустой клетки ровно 3 живых соседа - то на следующем ходу в ней зарождается жизнь (а иначе она остается пустой).

Так (рис.1), квадрат $2 \times 2$ («блок») остается неподвижным (у каждой его клетки по 3 живых соседа), линия из 3 клеток за 1 ход поворачивается на 90 градусов вокруг центра (поэтому ее называют «мигал-
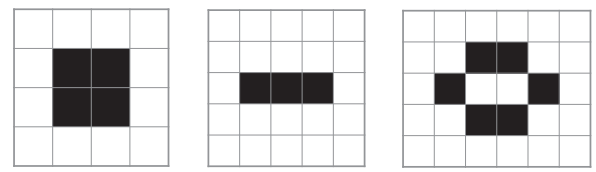

Рис. 1. Блок, семафор и улей

DOI: https://doi.org/10.4213/kvant20200502
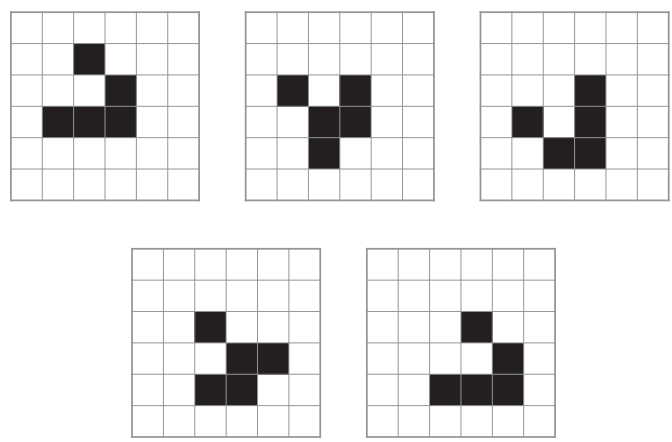

Рис. 2. Пять последовательных положений глайдера, летящего вправо-вниз

кой» или «семафором»), а прямоугольник $2 \times 3$ за один ход становится «ульем» (еще одной не меняющейся конфигурацией).

Слово «игра» тут не совсем точное, ибо все, на что влияет «игрок», это начальная расстановка клеток, все дальнейшее от него уже не зависит. Зато за жизнью такой «колонии клеток» бывает очень интересно наблюдать.

Глайдер (или планер), изображенный на рисунке 2, - конфигурация, которая движется вправо-вниз с постоянной скоростью под углом в 45 градусов. А другая невинно выглядящая конфигурация из пяти клеток - r-пентамино (рис.3) - эволюционирует на протяжении 1103 поколений. Глядя на r-пентамино, Конвей задал следующий интересный вопрос: бывают ли бесконечно растущие конфигурации? Он объявил награду в 50 долларов за решение этого вопроса - которую получил Билл Госпер, открывший глайдерное ружье, конфигурацию, постоянно «стреляющую» глайдерами.

Создание глайдерного ружья через какоето время позволило доказать, что игра «Жизнь» Тьюринг-полна: любую программу, которую можно запустить на какомнибудь компьютере, можно «эмулировать» с 

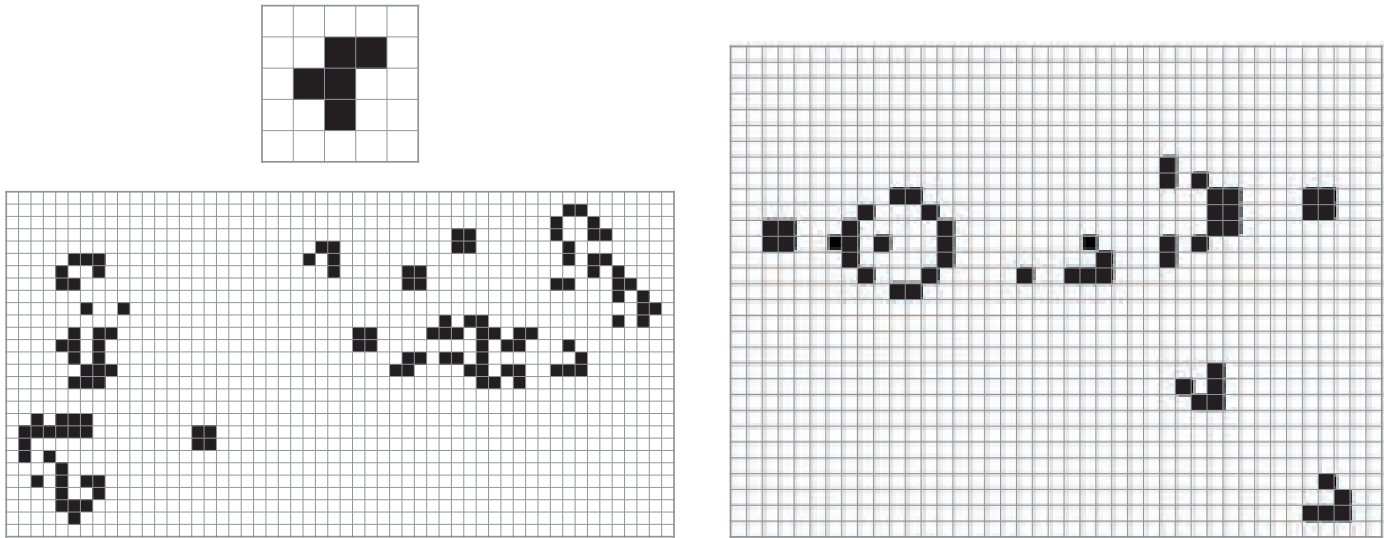

Рис. 3. Слева: r-пентамино (сверху исходное состояние, снизу конфигурация на 103-м шаге); справа. глайдерное ружье и три выпущенных им глайдера

помощью игры «Жизнь». Можно даже запустить игру «Жизнь» на игре «Жизнь» [1]!

Системы, живущие по аналогичным правилам - клетчатая плоскость, раскрашенная во много разных цветов-состояний, и правила перехода между этими состояниями, называют клеточными автоматами. Их рассматривали еще в 1950-е годы - они
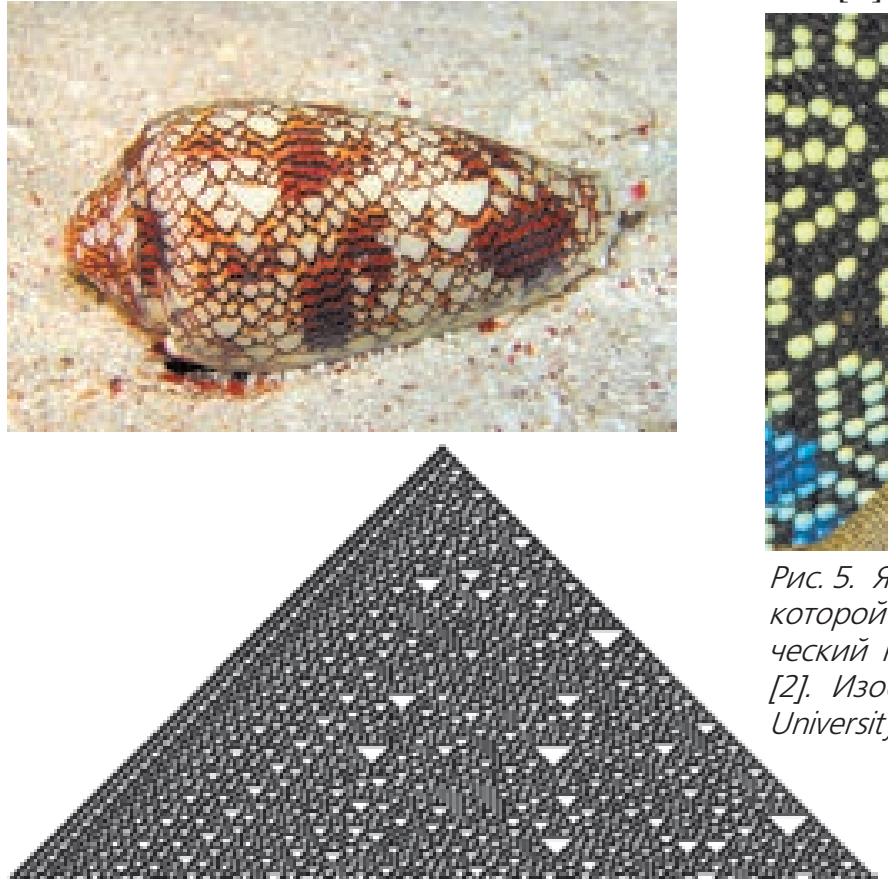

Рис. 4. Узор на ракушке (фото: R. Ling) и результат эволюции клеточного автомата "Правило 30», описанного С. ВольфраMOM восходят к идеям фон Неймана о самовоспроизводящихся машинах, и их использовали как упрощенные модели биологических процессов. Но не будет преувеличением сказать, что именно с игры «Жизнь» начался широкий расцвет клеточных автоматов. А их проявления в живой природе (рис.4,5) - предмет и недавних исследований [2].

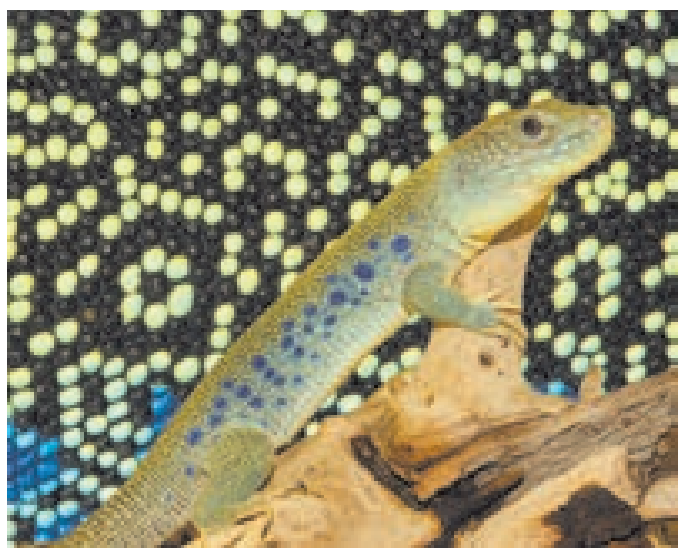

Рис. 5. Ящерица (Timon Lepidus), узору чешуек которой посвящена статья "Живой мезоскопический клеточный автомат из чешуек кожи» [2]. Изображение: M.Milinkovitch's laboratory, University of Geneva

\section{Конвей в общении}

Я много раз встречался с Конвеем на летних школах ISSMYS/ MOMISS в Бремене и в Лионе, и Конвей постоянно что-то рассказывал (как на фотографии на 


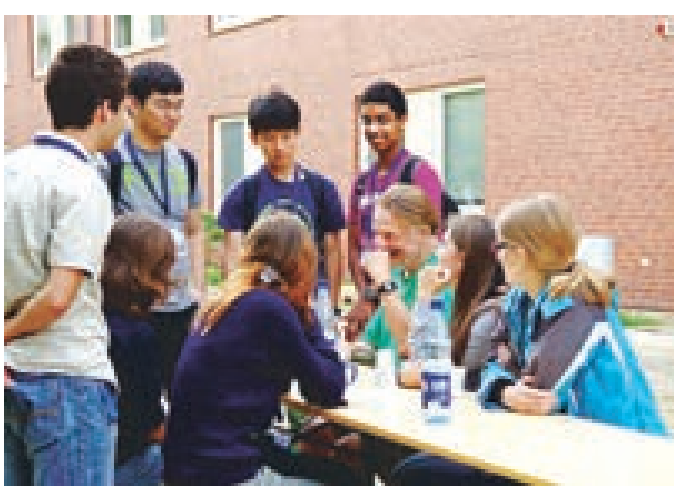

Рис. 6. Конвей в окружении участников летней школы. (Фото Рины Сергеевой)

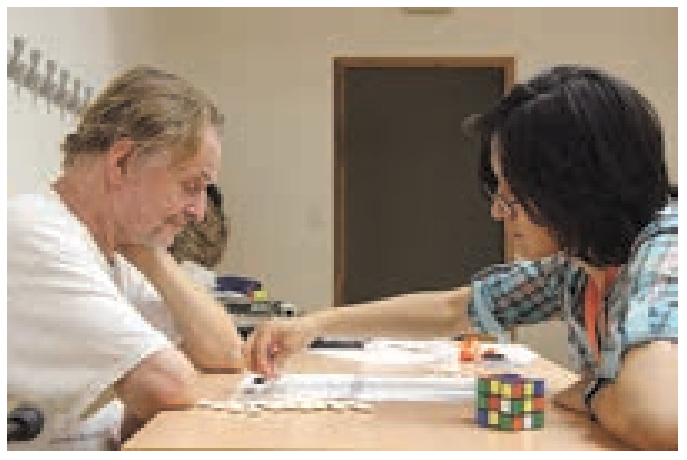

Рис. 7. Конвей играет в “футбол». (Фото Рины Сергеевой)

рисунке 6). От его историй невозможно было оторваться; это начиналось с утра и заканчивалось зачастую заполночь (конечно же, не исключая завтраков, обедов и ужинов!).

А на фотографии на рисунке 7 он играет с одной из участниц школы в изобретенную им настольную игру «футбол».

Известный математик Гил Калаи вспоминает про эту игру: «...Конвей установил для меня отдельное правило. Каждый раз, когда я был уверен, что проигрываю, мы менялись сторонами. Излишне уточнять, что это происходило более одного раза: я был уверен, что мое положение безнадежно, мы переворачивали доску, и вскоре после этого я опять был уверен, что мое положение безнадежно...»

А вот что пишет Теренс Тао (бывший тогда аспирантом Принстона, а ныне лауреат премии Филдса): «...Я все еще по- мню, как меня регулярно громил [Конвей] в этой игре, что было тогда полезным и необходимым уроком скромности для меня (как и для некоторых моих коллег-аспирантов)» .

\section{Игра «хакенбуш» и сюрреальные числа}

Одна из придуманных Конвеем игр «хакенбуш», или рубка кустарника. У нее довольно простые правила - но при этом она открывает дорогу к изобретенным им сюрреальным числам. Ее правила таковы: дан раскрашенный в два цвета граф, часть вершин которого расположена «на земле» (рис.8, слева). Игроки по
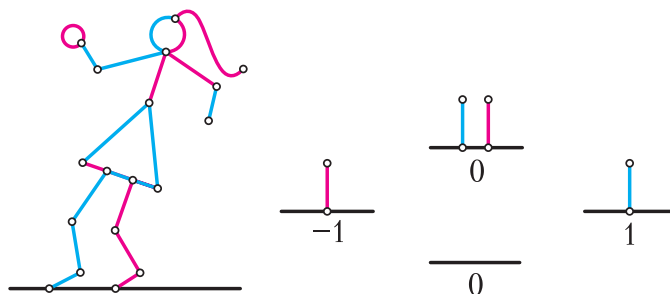

Рис. 8. Игра "хакенбуш»: пример игры и позиции со значениями -1, 0, 1

очереди перерезают ребра своего цвета. Если какая-то компонента графа более не связана с землей, то она удаляется; тот, кто не может сделать ход, проиграл.

Оказывается, что в этой игре ходить «невыгодно»: если, скажем, «Синий» игрок выигрывает начиная, то в той же начальной позиции он выигрывает и ходя вторым. И более того - каждой позиции можно сопоставить число, говорящее, «сколько у Синего свободных ходов». Если оно положительно, выигрывает Синий; если отрицательно - Красный. А если оно равно нулю, то в этой позиции начинающий (кем бы он ни был) всегда проигрывает.

Далее, если «сложить вместе» два графа (просто нарисовав их рядом) - то соответствующие числа тоже сложатся. Причем числа тут появляются не только целые, но и дробные. Так, графу из двух ребер, красному над синим (рис.9, слева), правильно сопоставить число $\frac{1}{2}$, потому что сумма двух его копий с позицией -1 будет 


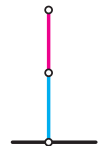

$1 / 2$
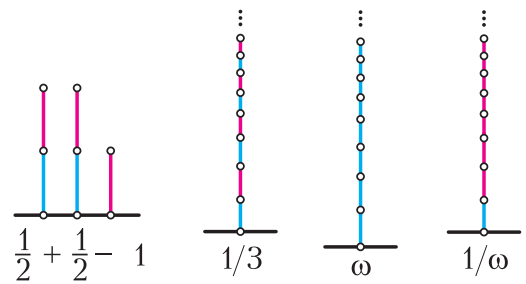

Рис.9. Позиция со значением 1/2 и уравнение для нее; бесконечный стебель со значением 1/3; два бесконечных стебля с не-вещественными значениями

нулевой (т.е. такой, где начинающий проигрывает).

Для конечных графов в игре «хакенбуш» сопоставляемые им числа всегда двоично-рациональные. Но - разрешив рассматривать бесконечные «стебли бамбука» (на которых, тем не менее, партия всегда завершается за конечное время), можно получить и позиции, которым сопоставляются любые вещественные числа.

Более того - оказывается, что «внутри теории игр» можно выбрать игры, которые можно объявить «числами». И естественно определить операции на них сложение, умножение и так далее. Однако среди таких чисел будут не только все вещественные, но и многие другие. Посмотрите на три правые картинки на рисунке 9. Вторая из этих трех игр, бесконечный синий «стебель бамбука», будет соответствовать «бесконечному числу» - ординалу $\omega$. А бесконечный стебель, в котором синее ребро только нижнее, - обратному к нему «числу» $1 / \omega$. И все это и есть придуманные Конвеем сюрреальные числа которые я здесь, впрочем, не определил. Об этом можно почитать в книге Пьера Деорнуа [3]; а еще сюрреальным числам (уже без связи с теорией игр) была посвящена статья [4] в «Кванте» аж в 1979 году.

Чтобы завершить рассказ об играх, можно упомянуть две книги Конвея - «On Numbers and Games» и написанную в соавторстве с Берлекемпом и Ги «Winning Ways for your Mathematical Plays». Обе стали бестселлерами сразу после выхода.

\section{Иррациональность корня из 2}

Мне хочется вспомнить и очень интересную - именно для школьников - статью
Конвея и Шипмана [5], где собраны разные доказательства иррациональности корня из 2 (и других квадратных корней). Это действительно интересное чтение: как один и тот же результат может получаться совершенно разными методами. Конечно же, там упоминается и классическое доказательство, в котором выясняется, что и числитель и знаменатель должны оказаться четными, - но не только оно.

Так, там появляется доказательство Танненбаума. А именно, если бы $\sqrt{2}$ был рационален, то можно было бы взять наименьшую пару целых чисел $(p, q)$ такую, что площадь квадрата со стороной $p$ равна сумме площадей двух квадратов со стороной $q$. Но поместив два таких квадрата внутрь квадрата со стороной $p$ (рис.10, слева), мы видим, что в таком случае площадь, закрытая дважды (а это квадрат со стороной $(2 q-p)$ ), равна непокрытой площади (а это два квадрата со стороной $(p-q)$ каждый). И мы получаем противоречие с минимальностью пары $(p, q)$.

А Конвей заметил, что аналогичным образом можно доказывать и иррациональность корня из 3 - оно изображено на рисунке 10 справа. ${ }^{1}$

Еще одно из собранных в этой же статье доказательств принадлежит Миклошу Лацковичу: если раскрыть скобки в выражении $(\sqrt{2}-1)^{n}$, то получится число вида $A \sqrt{2}-B$, где $A$ и $B$ - целые. И если бы $\sqrt{2}$
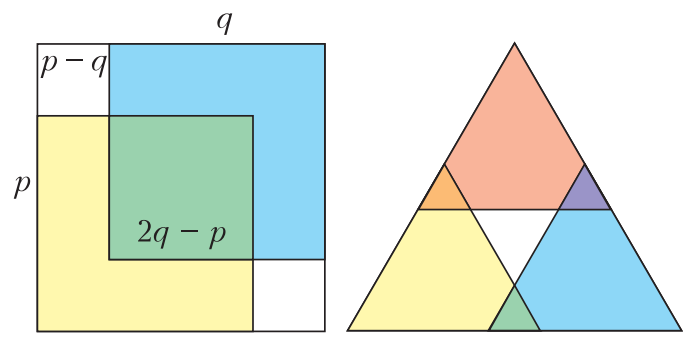

Рис. 10. Доказательство Танненбаума иррациональности $\sqrt{2}$ и его модификация Конвея для $\sqrt{3}$

${ }^{1}$ Есть и аналогичные доказательства для $\sqrt{5}$ и $\sqrt{6}$ : их можно найти в статье А.Спивака «Иррациональность корней из 2, 3,5 и 6» («Квант», 2010, №1). 
был рациональным числом вида $\frac{p}{q}$, то ни при каком $n$ такая величина не могла бы быть меньше $\frac{1}{q}$. И это противоречит тому,

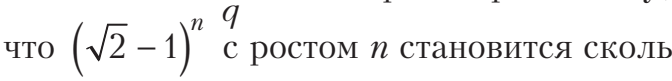
угодно малым.

\section{Головоломная задача}

Одна из не очень известных статей Конвея, совместная с М.С.Патерсоном, посвящена вот такой задаче. На доске написаны три числа: 6, 7 и 8. У Антуана, Боба и Чарли на шляпах написаны неотрицательные целые числа, сумма которых равна одному из трех чисел на доске; какому им неизвестно. Каждый из них видит числа двух других, но не свое.

Ведущий спрашивает по кругу у каждого из них, знает ли тот свое число. Требуется доказать, что рано или поздно ктонибудь ответит «да». Если на шляпах участников написаны числа 2, 2 и 3 соответственно, то перед этим прозвучит аж 18 ответов «нет» - что явно не помогает «лобовому» подходу к разбору случаев.

Теорема, которую изящным рассуждением доказали Конвей с Патерсоном в работе с говорящим названием «Задача, вызывающая головную боль» [6], утверждает, что если участников не меньше, чем вариантов чисел на доске, то рано или поздно ответ «да» прозвучит.

\section{Другое}

Многое и многое из того, чем занимался Конвей, осталось за кадром нашего рассказа. Это полином Конвея в топологии инвариант узла, позволяющий доказывать, что какие-то узлы нельзя развязать. Исследования решеток и кодов и три группы Конвея - исключительные простые группы. Мысленный эксперимент в квантовой механике - теорема о свободе воли. Оператор чтения [7] (итерации которого задают последовательность «Посмотри-и-скажu» $\left.{ }^{2}\right)$. Числовые фризы [8], которым была посвящена статья Конвея в «Кванте» (1991, №3) и вокруг которых происходит много интересного даже сегодня (см. статью Е.Смирнова в этом номере журнала). Книга «Квадратичные формы, данные нам в ощущениях», она переведена на русский язык. Атлас конечных групn, в создании которого Конвей участвовал, и сформулированная им и Нортоном гипотеза Monstrous Moonshine, относящаяся к самой большой спорадической простой группе - Монстру, и еще многое.

Но хочется завершить этот текст цитатой из Мартина Гарднера про формулу Конвея для вероятности выигрыша в игру Пенни (Penney's game): «Я не понимаю, почему это работает. Она просто выдает ответ как по волшебству, как многие другие алгоритмы Конвея». [9]

\section{Литература}

1. https://www.youtube.com/watch? $v=x P 5-$ iIeKXE8

2. L.Manukyan, S.Montandon, A.Fofonjka, S.Smirnov \& M. Milinkovitch. A living mesoscopic cellular automaton made of skin scales. - Nature, 544 (2017), pp. 173-179.

3. П.Деорнуа. Комбинаторная теория игр. М.: МЦНМО, 2017.

4. А. Кириллов, И. Клумова, А. Сосинский. Сюрреальные числа. - «Квант», 1979, №11.

5. J.H.Conway \& J.Shipman. Extreme Proofs I: The Irrationality of $\sqrt{2}$. - The Mathematical Intelligencer, 35 (2013), pp. 2-7.

6. J.H.Conway, M.S.Paterson \& Moscow (U.S.S.R.). A Headache-Causing Problem. - The American Mathematical Monthly, 127 (2020), pp.291-296.

7. J.H.Conway. The Weird and Wonderful Chemistry of Audioactive Decay. - Open Problems in Communication and Computation (1987), pp. 173-188.

8. J.H. Conway and H.S.M. Coxeter. Triangulated Polygons and Frieze Patterns. - The Mathematical Gazette, 57: 401 (Oct., 1973), pp.175183.

9. M. Gardner. On the paradoxical situations that arise from nontransitive relations. - Scientific American, 231: 4 (Oct., 1974), pp. 120-125.

\footnotetext{
${ }^{2}$ Вот она: 1, 11, 21, 1211, 111221, 312211, ..
} 